\title{
The comparison of the commonly used surrogates for baseline renal function in acute kidney injury diagnosis and staging
}

\author{
Charat Thongprayoon ${ }^{1 *}$, Wisit Cheungpasitporn' ${ }^{1}$ Andrew M. Harrison², Wonngarm Kittanamongkolchai ${ }^{1}$, \\ Patompong Ungprasert ${ }^{3}$, Narat Srivali ${ }^{4}$, Abbasali Akhoundi ${ }^{1}$ and Kianoush B. Kashani ${ }^{1,4}$
}

\begin{abstract}
Background: Baseline serum creatinine $(\mathrm{SCr})$ level is frequently not measured in clinical practice. The aim of this study was to investigate the effect of various methods of baseline SCr determination measurement on accuracy of acute kidney injury (AKI) diagnosis in critically ill patients.

Methods: This was a retrospective cohort study. All adult intensive care unit (ICU) patients admitted at a tertiary referral hospital from January 1, 2011 through December 31, 2011, with at least one measured SCr value during ICU stay, were included in this study. The baseline $\mathrm{SCr}$ was considered either an admission $\mathrm{SCr}\left(\mathrm{SCr}_{\mathrm{ADM}}\right)$ or an estimated $\mathrm{SCr}$, using MDRD formula, based on an assumed glomerular filtration rate (GFR) of $75 \mathrm{ml} / \mathrm{min} / 1.73 \mathrm{~m}^{2}\left(\mathrm{SCr}_{\mathrm{GFR}-75}\right.$ ). Determination of AKI was based on the KDIGO SCr criterion. Propensity score to predict the likelihood of missing SCr was used to generate a simulated cohort of 3566 patients with baseline outpatient SCr, who had similar characteristics with patients whose outpatient SCr was not available.
\end{abstract}

Results: Of 7772 patients, 3504 (45.1\%) did not have baseline outpatient SCr. Among patients without baseline outpatient SCr, AKI was detected in 571 (16.3\%) using the $\operatorname{SCr}_{\text {ADM }}$ and 997 (28.4\%) using $\operatorname{SCr}_{\text {GFR-75 }}(p<.001)$. Compared with non-AKI patients, patients who met AKI only by SCr $\mathrm{SDM}_{\text {, }}$, but not $\mathrm{SCr}_{\mathrm{GFR}-75}$, were significantly associated with 60-day mortality (OR 2.90; $95 \%$ Cl 1.66-4.87), whereas patients who met AKI only by $\mathrm{SCr}_{\text {GFR-75, }}$ but not $\mathrm{SCr}_{\mathrm{ADM}}$, had a non-significant increase in 60-day mortality risk (OR 1.33; $95 \% \mathrm{Cl}$ 0.94-1.88). In a simulated cohort of patients with baseline outpatient SCr, SCr GFR-75 yielded a higher sensitivity (77.2 vs. $50.5 \%$ ) and lower specificity (87.8 vs. $94.8 \%$ ) for the AKI diagnosis in comparison with $\mathrm{SCr}_{\mathrm{ADM}}$.

Conclusions: When baseline outpatient SCr was not available, using $\mathrm{SCr}_{\text {GFR-75 }}$ as surrogate for baseline SCr was found to be more sensitive but less specific for AKI diagnosis compared with using $\mathrm{SCr}_{\mathrm{ADM}}$. This resulted in higher incidence of AKI with larger likelihood of false-positive cases.

Keyword: Acute kidney injury, Back-calculation, Creatinine, Estimated creatinine, Mortality

\section{Background}

Acute kidney injury (AKI) is commonly associated with high morbidity and mortality in critically ill patients [1-4]. Even a modest degree of AKI is associated with mortality, morbidity, and increased healthcare cost [5-10]. The Kidney Disease: Improving Global Outcomes (KDIGO) criteria was developed and validated to standardize the diagnosis

\footnotetext{
* Correspondence: charat.thongprayoon@gmail.com

'Division of Nephrology and Hypertension, Mayo Clinic, 200 First Street SW, Rochester, MN 55905, USA

Full list of author information is available at the end of the article
}

and severity of AKI based on absolute or relative increases from baseline serum creatinine ( $\mathrm{SCr}$ ) levels, as well as progressive oliguria [11]. Thus, determination of baseline $\mathrm{SCr}$ is important to diagnose and classify AKI $[12,13]$.

Despite development of the KDIGO criteria, classification of AKI remains challenging, as baseline outpatient $\mathrm{SCr}$ measurement-a marker of kidney function prior to the critical illness-is often unavailable [14]. In this case, the Acute Dialysis Quality Initiative (ADQI) has recommended backward calculation of baseline $\mathrm{SCr}$ using the Modification of Diet in Renal Disease (MDRD) formula, 
this formula assumes an estimated glomerular filtration rate (eGFR) value of $75 \mathrm{ml} / \mathrm{min} / 1.73 \mathrm{~m}^{2}\left(\mathrm{SCr}_{\mathrm{GFR}-75}\right)$ for all patients with missing data [15]. However, backward calculation can lead to misclassification of AKI, particularly in the early stages of this syndrome $[16,17]$.

The European Renal Best Practice (ERBP) recently proposed using the first documented $\mathrm{SCr}$ value on hospital admission $\left(\mathrm{SCr}_{\mathrm{ADM}}\right)$-rather than $\mathrm{SCr}_{\mathrm{GFR}-75}$-as the baseline $\mathrm{SCr}$ when baseline outpatient $\mathrm{SCr}$ measurements are missing [18]. However, the use of $\mathrm{SCr}_{\mathrm{ADM}}$ as the baseline $\mathrm{SCr}$ can be inaccurate in patients with communityacquired $\mathrm{AKI}$, as the $\mathrm{SCr}$ may have already increased prior to hospitalization $[19,20]$. Additionally, the predictive performance of these two methods on mortality has not been well studied [18].

The primary objective of this study was to compare the incidence and staging of AKI according to $\mathrm{SCr}$ criteria using $\mathrm{SCr}_{\mathrm{GFR}-75}$ versus $\mathrm{SCr}_{\mathrm{ADM}}$. The secondary objective of this study was to determine the accuracy of AKI diagnosis in critically ill patients using $\mathrm{SCr}$ estimation, based on assumed GFR and $\mathrm{SCr}_{\mathrm{ADM}}$, compared to the reference standard of $\mathrm{SCr}$ measurement.

\section{Methods}

\section{Study Population}

This is a single-center retrospective study conducted at a tertiary referral hospital. We studied all adult patients (age $\geq 18$ years) admitted to the ICU at Mayo Clinic in Rochester, MN, from January 1, 2011 through December 31, 2011. We included patients who had at least one SCr measured during the ICU admission. Patients with a history of stage 5 chronic kidney disease (CKD) or end-stage renal disease (ESRD), patients who received any dialysis modalities within 14 days prior to the ICU admission, and those who did not provide research authorization were excluded from the study. Stage 5 CKD and ESRD were identified based on ICD-9 code assignment (Additional file 1: Table S1) or baseline outpatient $\mathrm{SCr}$-calculated eGFR of $<15 \mathrm{ml} / \mathrm{min} / 1.73 \mathrm{~m}^{2}$. For patients with multiple ICU admissions, only the first ICU admission during the study period was included in the analysis. This study was approved by the Mayo Clinic institutional review board.

We divided eligible patients into two groups, based on the availability of outpatient $\mathrm{SCr}$ between 365 and 7 days prior to hospital admission (Fig. 1). One group included patients without baseline outpatient $\mathrm{SCr}(n=3504)$. This is the cohort of patients for whom the use of surrogates for baseline $\mathrm{SCr}$ was actually applied. This cohort was used to compare the incidence and outcomes of AKI using $\mathrm{SCr}_{\mathrm{GFR}-75}$ versus $\mathrm{SCr}_{\mathrm{ADM}}$ in order to represent the real-world finding. The second group included patients with baseline outpatient SCr $(n=4268)$.

\section{Simulated cohort}

AKI diagnosis based on outpatient $\mathrm{SCr}$ baseline can be used as a reference standard to assess the sensitivity and specificity of AKI diagnosis using various surrogate estimates. However, due to significant differences in clinical characteristics between patients with and without outpatient SCr (Table 1), the finding in the cohort of patients with outpatient $\mathrm{SCr}$ might not be generalizable to patients without outpatient SCr. Therefore, we developed a propensity score model in which the outcome was whether baseline SCr was missing $(n=3504)$ or not

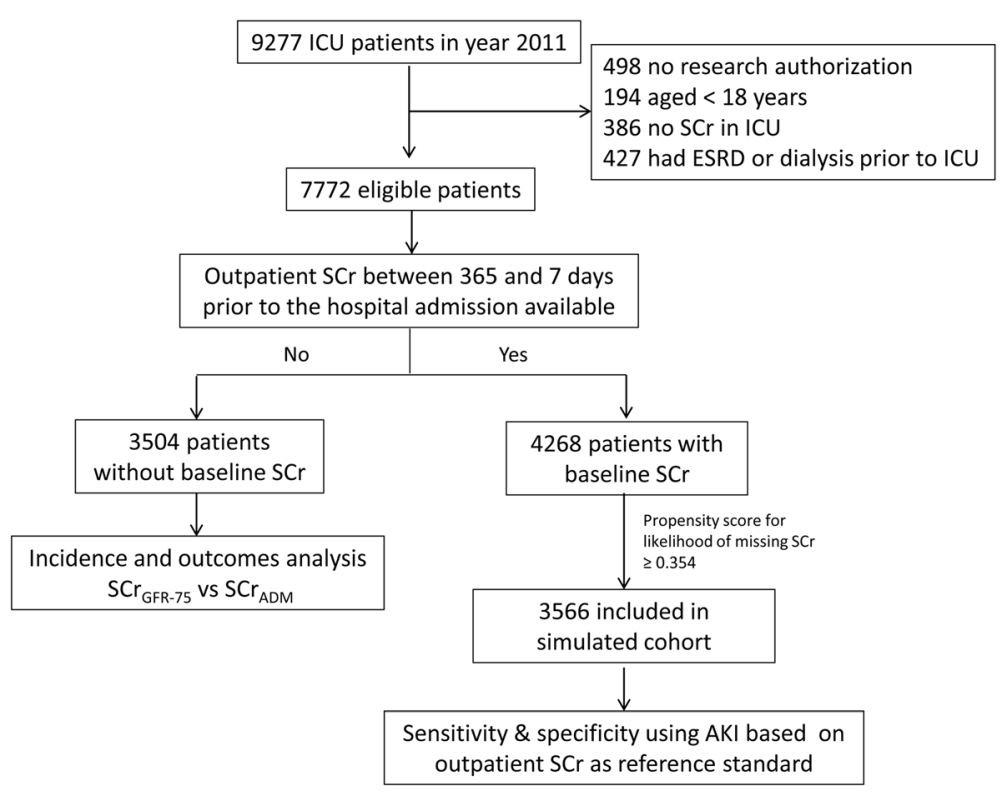

Fig. 1 Study inclusion and exclusion flow diagram 
Table 1 Clinical characteristics and outcomes of eligible critically ill patients admitted to the ICU during the study period

\begin{tabular}{|c|c|c|c|c|c|}
\hline Characteristics & Outpatient SCr not available & Outpatient $\mathrm{SCr}$ available & $p^{*}$ & Simulated Cohort ${ }^{a}$ & $p^{*}$ \\
\hline $\bar{N}$ & 3,504 & 4,268 & & 3566 & \\
\hline Age, year & $62 \pm 18$ & $65 \pm 16$ & $<0.001$ & $63 \pm 16$ & 0.05 \\
\hline Male sex & $2013(57)$ & $2508(59)$ & 0.24 & $2033(57)$ & 0.71 \\
\hline White & $3246(93)$ & 4039 (95) & $<0.001$ & $3347(94)$ & 0.10 \\
\hline \multicolumn{6}{|l|}{ Baseline creatinine, mg/dL } \\
\hline - Admission SCr & $1.1 \pm 0.9$ & $1.1 \pm 0.8$ & 0.55 & $1.1 \pm 0.8$ & 0.10 \\
\hline - Single imputation of GFR $75 \mathrm{ml} / \mathrm{min} / 1.73 \mathrm{~m}^{2}$ & $1.0 \pm 0.1$ & $1.0 \pm 0.1$ & 0.20 & $1.0 \pm 0.1$ & 0.06 \\
\hline - Baseline outpatient SCr & - & $1.1 \pm 0.4$ & - & $1.1 \pm 0.4$ & - \\
\hline \multicolumn{6}{|l|}{ Comorbidities } \\
\hline - Diabetes Mellitus & $723(21)$ & $1114(26)$ & $<0.001$ & $730(20)$ & 0.87 \\
\hline - Hypertension & $1836(52)$ & $2653(62)$ & $<0.001$ & $2004(56)$ & 0.04 \\
\hline - Coronary artery disease & $809(23)$ & $1376(32)$ & $<0.001$ & $793(22)$ & 0.39 \\
\hline - Stroke & $272(8)$ & $472(11)$ & $<0.001$ & $240(7)$ & 0.12 \\
\hline - Peripheral vascular disease & $120(3)$ & $258(6)$ & $<0.001$ & $60(2)$ & 0.10 \\
\hline - Congestive heart failure & $311(9)$ & $588(14)$ & $<0.001$ & $252(7)$ & 0.07 \\
\hline Medical ICU type & $1733(49)$ & $2036(48)$ & 0.12 & $1689(47)$ & 0.08 \\
\hline APACHE III & $44 \pm 22$ & $50 \pm 20$ & $<0.001$ & $46 \pm 18$ & 0.06 \\
\hline Renal replacement therapy use in ICU & $89(3)$ & $109(3)$ & 0.97 & $78(2)$ & 0.33 \\
\hline ICU mortality & $102(3)$ & $136(3)$ & 0.48 & $94(3)$ & 0.48 \\
\hline 60-day mortality & $298(9)$ & $468(11)$ & $<0.001$ & $338(9)$ & 0.15 \\
\hline
\end{tabular}

Abbreviations: APACHE Acute Physiology and Chronic Health Evaluation, GFR glomerular filtration rate, ICU intensive care unit, IQR interquartile range SCr serum creatinine, SOFA sequential organ failure assessment

*Compared with patients without baseline outpatient $\mathrm{SCr}$

${ }^{\mathrm{a}}$ Simulated cohort was a subset of patients with available baseline outpatient $\mathrm{SCr}$ with propensity score to predict the likelihood of missing baseline SCr of $\geq 0.354$ Continuous data are presented as mean \pm SD, categorical data are presented as $\mathrm{N}(\%)$, if not indicated

$(n=4268)$ to predict the likelihood of missing baseline SCr. The propensity model included age, race, diabetes mellitus, hypertension, coronary artery disease, stroke, peripheral vascular disease, congestive heart failure, and APACHE III at ICU admission as covariates. We applied this model to the group of patients with baseline outpatient $\mathrm{SCr}$ and then selected patients with a propensity score of $\geq 0.354$ (post-hoc cut-off) to generate a study cohort of patients with similar characteristics to patients whose baseline $\mathrm{SCr}$ was not available, while preserving known baseline SCr $(n=3566)$. We used this simulated cohort to analyze the sensitivity and specificity of AKI diagnosis based on different surrogates. AKI diagnosis based on outpatient SCr was used as the reference standard.

\section{Data collection}

Clinical characteristics, demographic information, and laboratory data were collected using manual and automated retrieval from the institutional electronic medical records. We utilized data from the Mayo Clinic Life Science System (MCLSS) and the Multidisciplinary Epidemiology and Translational Research in Intensive Care (METRIC) database. The MCLSS database contained demographic characteristics, clinical data, hospital admission information, diagnosis codes, procedure codes, laboratory test results, and flowsheet data of both in- and outpatients at our institution [21]. The METRIC database contained ICU admission information, pertinent vital signs, fluid input/output, and medication administration record data of all patients admitted in ICU at our institution [22]. Data variables collected included age, sex, race, known comorbidities at hospital admission, ICU type, severity of illness at ICU admission, ICU length of stay, and ICU discharge status. SCr measurements were collected for each eligible patient up to one year prior to ICU admission. The baseline outpatient $\mathrm{SCr}$ was defined as the most recent outpatient $\mathrm{SCr}$ measured between 365 and 7 days prior to the hospital admission. The eGFR was derived using the MDRD equation, with CKD being defined as a calculated eGFR of $<60 \mathrm{ml} /$ $\min / 1.73 \mathrm{~m}^{2}$. We identified comorbidities from clinical notes in electronic medical record using a validated electronic note search strategy [21]. The severity of illness at ICU admission was evaluated using the Acute Physiology and Chronic Health Evaluation (APACHE) III score [23].

\section{AKI diagnosis and classification}

We identified and staged AKI based solely on the $\mathrm{SCr}$ criterion of the KDIGO definition [11]. AKI was defined 
as an increase in $\mathrm{SCr}$ in the ICU of $\geq 0.3 \mathrm{mg} / \mathrm{dL}$ or relative change of $\geq 50 \%$ from the baseline. The baseline $\mathrm{SCr}$ was calculated using two different methods; 1) the first $\mathrm{SCr}$ available during hospital admission $\left(\mathrm{SCr}_{\mathrm{ADM}}\right)$, and 2) an estimated SCr using the MDRD formula, based on an assumed GFR of $75 \mathrm{ml} / \mathrm{min} / 1.73 \mathrm{~m}^{2}$ ( $\mathrm{SCr}_{\mathrm{GFR}-75}$, as recommended by the ADQI working group). We used the following equation for backward calculation of $\mathrm{SCr}_{\mathrm{GFR}-75}$ :

$$
\begin{aligned}
\operatorname{SCr}_{\mathrm{GFR}-75}= & \left(75 /\left[186 *\left(\text { age }^{-0.203}\right) *(0.742 \text { for women })\right.\right. \\
& *(1.21 \text { for black })])-0.887
\end{aligned}
$$

\section{Clinical outcomes}

The primary outcome was all-cause mortality at 60 days following ICU admission. We reviewed patient vital statistics by reviewing the patient registration and electronic medical records. In patients whose vital status at 60 days after ICU admission was unknown, the Social Security Death Index was used [24].

\section{Statistical analysis}

Continuous variables were reported as mean with standard deviation (SD) or median with interquartile range (IQR), as appropriate. All categorical variables were reported as counts with percentages. The difference in the AKI diagnosis using $\mathrm{SCr}_{\mathrm{ADM}}$ andSCr $\mathrm{G}_{\mathrm{GFR}-75}$ was assessed using McNemar's test. The agreement of AKI diagnosis and staging, based on $\mathrm{SCr}_{\mathrm{ADM}}$ and $\mathrm{SCr}_{\mathrm{GFR}-75}$, was assessed using Cohen's weighted kappa coefficient with linear weight between AKI stages. According to the results of AKI diagnosis, based on $\mathrm{SCr}_{\mathrm{ADM}}$ and $\mathrm{SCr}_{\mathrm{GFR}-75}$, we classified patients into 4 groups: (1) patients who had AKI regardless of baseline SCr calculation method, (2) patients who had AKI based only on $\mathrm{SCr}_{\mathrm{ADM}}$, (3) patients who had AKI only based on $\mathrm{SCr}_{\mathrm{GFR}-75}$, and (4) patients who did not have AKI, regardless of baseline $\mathrm{SCr}$ determination methodology. We adjusted the odds ratio (OR) for age, ICU type, and APACHE III scores to assess 60-day mortality for the first three groups, using the fourth group as the reference group. The association between AKI stages and 60-day mortality was assessed using a logistic regression analysis. The predictive performance of the $\mathrm{SCr}$ criterion, using $\mathrm{SCr}_{\mathrm{ADM}}$ and $\mathrm{SCr}_{\mathrm{GFR}-75}$, for 60-day mortality was assessed by C-statistics, after which we compared their performances using Delong's test. We calculated net reclassification improvement to evaluate how using $\mathrm{SCr}_{\mathrm{GFR}}$ 75 as baseline renal function for AKI diagnosis changed risk classification for 60-day mortality.

Sensitivities and specificities were compared using McNemar's test. To determine the optimal GFR used for $\mathrm{SCr}$ estimation, we estimated based on an assumed GFR with an increment of $5 \mathrm{ml} / \mathrm{min} / 1.73 \mathrm{~m}^{2}$, ranging from
30 to $120 \mathrm{ml} / \mathrm{min} / 1.73 \mathrm{~m}^{2}$. The sensitivity and specificity of AKI diagnosis, according to the estimated $\mathrm{SCr}$ of each assumed GFR, was calculated using AKI diagnosis according to the baseline outpatient $\mathrm{SCr}$ as a reference standard. The Youden index, which yielded the highest sum of sensitivity and specificity, was used to identify the optimal GFR used for $\mathrm{SCr}$ estimation. The subgroup analyses, based on sex, age, and the presence of abnormal renal function at presentation, were performed to investigate the optimal GFR used in each subgroup. A two-sided $p$ value of less than 0.05 was considered statistically significant. All analyses were performed using JMP statistical software (version 10.0, SAS, Cary, NC).

\section{Results}

During the study period, 9277 critically ill patients were admitted to the ICU. Of these, 1,505 were excluded: 498 did not provide authorization to use their data for research, 194 aged $<18$ years, and 386 had no measured $\mathrm{SCr}$ values in ICU, 427 had ESRD, or received dialysis within 14 days prior to ICU admission. Thus, 7772 patients were included in this study. The baseline outpatient SCr was not available for 3504 of these patients (45.1\%). The clinical characteristics of these patients upon ICU admission and their outcomes are summarized in Table 1. Patients who had available baseline outpatient SCr were older, Caucasian, had more known comorbidities, and had higher APACHE and SOFA scores at ICU admission. Patients in the simulated cohort had similar characteristics to patients without baseline outpatient SCr.

\section{AKI diagnosis and staging using the admission and estimated $\mathrm{SCr}$}

Among patients without baseline outpatient $\mathrm{SCr}$, using $\mathrm{SCr}_{\mathrm{ADM}}$, AKI occurred in 571 patients $(16.3 \%)$, with $12.1 \%$ in stage $1,2.3 \%$ in stage 2 , and $1.9 \%$ in stage 3 . UsingSCr $\mathrm{GFR}_{\text {GF-75, }}$ AKI occurred in 997 patients $(28.4 \%)$, with $15.6 \%$ in stage $1,7.4 \%$ in stage 2 and $5.5 \%$ in stage 3. $\mathrm{SCr}_{\mathrm{GFR}-75}$ classified more patients into AKI than $\operatorname{SCr}_{\mathrm{ADM}}(p<.001)$ (Table 2).

The percentage agreement for AKI diagnosis using $\mathrm{SCr}_{\mathrm{ADM}}$ and $\mathrm{SCr}_{\mathrm{GFR}-75}$ for baseline $\mathrm{SCr}$ estimation was $79.5 \%$ with a kappa of 0.42 (95 \% CI, 0.39-0.46). $\mathrm{SCr}_{\mathrm{ADM}}$ and $\mathrm{SCr}_{\mathrm{GFR}-75}$ as baseline $\mathrm{SCr}$ agreed in $425 \mathrm{AKI}$ cases and 2361 non-AKI cases. Using $\mathrm{SCr}_{\mathrm{ADM}}$ and $\mathrm{SCr}_{\mathrm{GFR}-75}$ resulted in a discrepancy in AKI diagnoses of 718 cases (20.5\%). 146 patients met the AKI diagnosis by only $\mathrm{SCr}_{\mathrm{ADM}}$ and 572 met the AKI diagnosis using only $\mathrm{SCr}_{\mathrm{GFR}-75}$. The percentage agreement for AKI staging, using both $\mathrm{SCr}_{\mathrm{ADM}}$ and $\mathrm{SCr}_{\mathrm{GFR}-75}$, was $74.4 \%$ with a kappa of 0.39 (95\% CI, $0.36-0.42)$. Ninety six percent of AKI based only by $\mathrm{SCr}_{\mathrm{GFR}-75}$ but not $\mathrm{SCr}_{\mathrm{ADM}}$ occurred within 24 hours of ICU admission. 
Table 2 AKI diagnoses and staging using admission $\mathrm{SCr}$ and GFR-estimated SCr for patients without baseline outpatient $\mathrm{SCr}$ $(n=3504)$

\begin{tabular}{|c|c|c|c|c|c|}
\hline \multirow{2}{*}{$\begin{array}{l}\text { AKI stage } \\
\left(\mathrm{SCr}_{\mathrm{GFR}-75}\right)\end{array}$} & \multicolumn{4}{|c|}{ AKI stage $\left(\mathrm{SCr}_{\mathrm{ADM}}\right)$} & \multirow{2}{*}{$\begin{array}{l}\text { Total N } \\
\text { (\%) }\end{array}$} \\
\hline & 0 & 1 & 2 & 3 & \\
\hline 0 & $2361(67.4)$ & $133(3.8)$ & $13(0.4)$ & $0(0)$ & 2507 (71.6) \\
\hline 1 & $356(10.2)$ & $160(4.6)$ & $28(0.8)$ & $2(0.1)$ & $546(15.6)$ \\
\hline 2 & $134(3.8)$ & $85(2.4)$ & $31(0.9)$ & $10(0.3)$ & $260(7.4)$ \\
\hline 3 & $82(2.3)$ & $45(1.3)$ & $9(0.3)$ & $55(1.6)$ & $191(5.5)$ \\
\hline Total, N (\%) & 2933 (83.7) & $423(12.1)$ & $81(2.3)$ & $67(1.9)$ & 3504 (100) \\
\hline
\end{tabular}

Kappa $=0.42(95 \% \mathrm{Cl} 0.39-0.46)$ and percentage agreement $=79.5 \%$ for AKI diagnosis,

Kappa $=0.39(95 \% \mathrm{Cl} 0.36-0.42)$ and percentage agreement $=74.4 \%$ for AKI staging

Abbreviation: AKI, acute kidney injury; $\mathrm{SCr}_{\mathrm{ADM}}$, the admission serum creatinine; $\mathrm{SCr}_{\mathrm{GFR}-75}$, an estimated serum creatinine based on an assumed GFR of $75 \mathrm{ml} / \mathrm{min} / 1.73 \mathrm{~m}^{2}$

\section{Risk for 60-day mortality}

Of the total cohort, $8.5 \%(N=298)$ died within 60 days of ICU admission. The 60-day mortality rates after ICU admission for AKI stages by using $\mathrm{SCr}_{\mathrm{ADM}}$ and $\mathrm{SCr}_{\mathrm{GFR}-75}$ are shown in Fig. 2. Compared with patients without AKI, patients who met AKI regardless of baseline SCr methodology, and patients who met AKI only by $\mathrm{SCr}_{\mathrm{ADM}}$, but not $\mathrm{SCr}_{\mathrm{GFR}-75}$, were significantly associated with increased 60-day mortality ( $\mathrm{OR}=3.66$ [95 \% CI, 2.65-5.04] and $\mathrm{OR}=2.90$ [95\% CI, 1.66-4.87]). However, patients who met AKI only by $\mathrm{SCr}_{\mathrm{GFR}-75}$, but not $\mathrm{SCr}_{\mathrm{ADM}}$, had a non-significant increase in 60-day mortality risk (OR 1.33; 95 \% CI 0.94-1.88) (Table 3 and Fig. 3). Calculating the performance for prediction of 60-day mortality, the C-statistic for AKI stages using $\mathrm{SCr}_{\mathrm{ADM}}$ and $\mathrm{SCr}_{\mathrm{GFR}-75}$ as baseline $\mathrm{SCr}$ were 0.64 and 0.68 respectively $(p=.001)$. Using $\mathrm{SCr}_{\mathrm{GFR}-75}$ for AKI diagnosis improved risk classification for 60-day mortality with net reclassification improvement of $4.7 \%$.

\section{Sensitivity and specificity for AKI diagnosis}

In the simulated cohort of patients with available baseline outpatient $\mathrm{SCr}$, the mean baseline $\mathrm{SCr}$ (SD) was $1.1 \pm$ $0.4 \mathrm{mg} / \mathrm{dL}$. Of these patients, $20.7 \%(n=738)$ experienced AKI using known baseline outpatient SCr, $14.6 \%(n=520)$ using $\mathrm{SCr}_{\mathrm{ADM}}$, and $25.7 \%(n=915)$ using $\mathrm{SCr}_{\mathrm{GFR}-75}$. Application of $\mathrm{SCr}_{\mathrm{GFR}-75}$ as baseline $\mathrm{SCr}$ yielded a sensitivity of $77.2 \%$ and specificity of $87.8 \%$ for the AKI diagnosis. Using $\mathrm{SCr}_{\mathrm{ADM}}$ as baseline $\mathrm{SCr}$ yielded a sensitivity of $50.5 \%$ and specificity of $94.8 \%$. Overall, $\mathrm{SCr}_{\mathrm{GFR}-75}$ was more sensitive $(p<.001)$, whereas $\mathrm{SCr}_{\mathrm{ADM}}$ was found to be more specific $(p<.001)$. The application of either $\mathrm{SCr}_{\text {GFR-75 }}$ or $\mathrm{SCr}_{\mathrm{ADM}}$, compared to AKI diagnosis and staging based on baseline outpatient $\mathrm{SCr}$, resulted in bi-directional misclassification of AKI (Additional file 1: Table S2).

\section{Optimal GFR used for SCr estimation}

The sensitivity and specificity of AKI diagnosis, when using different assumed GFR for SCr estimation, were shown in Table 4. The most optimal GFR with the highest sum of sensitivity and specificity was $85 \mathrm{ml} / \mathrm{min} / 1.73 \mathrm{~m}^{2}$, yielding a sensitivity of $85.2 \%$ and a specificity of $83.0 \%$. The sensitivity and specificity in different subgroups based on sex, age, and the presence of abnormal renal function at presentation are summarized in Additional file 1: Table S3 with the most optimal GFR for SCr estimation indicated. The application of $\mathrm{SCr}_{\mathrm{ADM}}$ for AKI diagnosis in patients with abnormal renal function at presentation resulted in significantly lower sensitivity (41.7 vs. $65 \%$ ), but similar specificity (92.7 vs. $95.3 \%$ ), for AKI diagnosis.

\section{Discussion}

We conducted a retrospective cohort study to assess the effect of using different baseline SCr methods, $\mathrm{SCr}_{\mathrm{GFR}-75}$ and $\mathrm{SCr}_{\mathrm{ADM}}$, on the incidence of $\mathrm{AKI}$ as well as prognostication

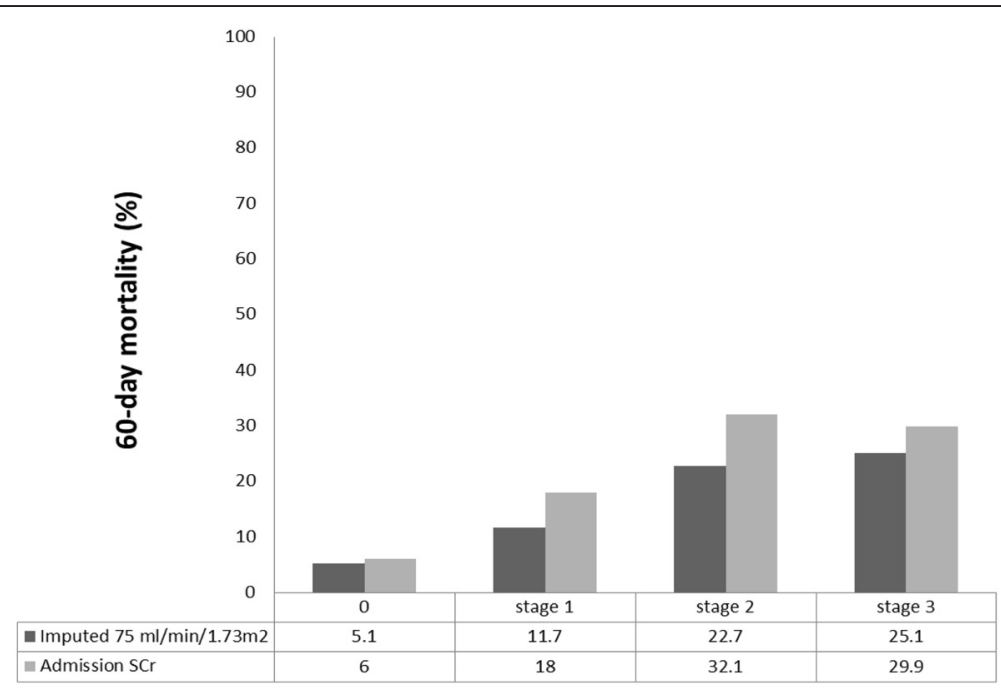

Fig. 2 60-day mortality risk stratified by AKI stage in patients without baseline outpatient $\mathrm{SCr}$ 
Table 3 60-day mortality risk based on the AKI diagnosis using admission and GFR-estimated SCr in patients without baseline outpatient SCr $(n=3504)$

\begin{tabular}{|c|c|c|}
\hline $\begin{array}{l}\text { N 60-day mortality, } \\
\text { n (\%) }{ }^{a} \text { OR ( } 95 \% \text { Cl) }\end{array}$ & No AKI-SCr & $\mathrm{AKI}-\mathrm{SCr}_{\mathrm{ADM}}$ \\
\hline \multirow[t]{3}{*}{ No AKI- SCr GFR-75 } & 2361 & 146 \\
\hline & $4.5 \%$ & $13.7 \%$ \\
\hline & 1 (ref) & $2.90(1.66-4.87)$ \\
\hline \multirow[t]{3}{*}{ AKI- SCr ${ }_{\text {GFR-75 }}$} & 572 & 425 \\
\hline & $12.1 \%$ & $24.0 \%$ \\
\hline & $1.33(0.94-1.88)$ & $3.66(2.65-5.04)$ \\
\hline
\end{tabular}

Abbreviation: $A K I$ acute kidney injury, $\mathrm{Cl}$ confidence interval, $\mathrm{SCr}_{A D M}$ the admission serum creatinine, $\mathrm{SCr}_{G F R-75}$ an estimated serum creatinine based on an assumed GFR of $75 \mathrm{ml} / \mathrm{min} / 1.73 \mathrm{~m}^{2}$

adjusted for age, APACHE III score and ICU type

performance of the KDIGO definition. We demonstrated that using $\mathrm{SCr}_{\mathrm{GFR}-75}$ as a baseline $\mathrm{SCr}$ for AKI diagnosis was associated with the larger number of classified AKI cases. However, using $\mathrm{SCr}_{\mathrm{GFR}-75}$ resulted in the misclassification of non-AKI cases into AKI, as AKI cases only by $\mathrm{SCr}_{\mathrm{GFR}-75}$, but not $\mathrm{SCr}_{\mathrm{ADM}}$, had a no significant increase in 60-day mortality risk. This finding is consistent with SCr $r_{\text {GFR-75 }}$ as a more sensitive and $\mathrm{SCr}_{\mathrm{ADM}}$ as a more specific surrogate for AKI diagnosis when compared with outpatient $\mathrm{SCr}$ as the reference standard.

Ideally, outpatient $\mathrm{SCr}$ values, which are reflective of patient pre-morbid kidney function, should be used as the baseline SCr for AKI diagnosis [25]. However, the lack of baseline outpatient $\mathrm{SCr}$ level is a common problem encountered in clinical practice $[25,26]$. Following the ADQI statement, the use of $\mathrm{SCr}_{\mathrm{GFR}-75}$ as a baseline $\mathrm{SCr}$ has been shown to lead to misclassification of AKI in ICU patients
$[16,17]$ and following cardiac surgery [27]. The European Renal Best Practice (ERBP) has recommended the use of $\mathrm{SCr}_{\mathrm{ADM}}$, rather than $\mathrm{SCr}_{\mathrm{GFR}-75}$, when baseline outpatient $\mathrm{SCr}$ measurements are not available [18]. In our study, we demonstrated that using either $\mathrm{SCr}_{\mathrm{GFR}-75}$ or $\mathrm{SCr}_{\mathrm{ADM}}$ led to misclassification of AKI diagnosis and staging. Use of $\mathrm{SCr}_{\mathrm{GFR}-75}$ has been shown to inflate AKI incidence, whereas use of $\mathrm{SCr}_{\mathrm{ADM}}$ underestimates $\mathrm{AKI}$ incidence $[14,16,17]$. The inflation of AKI diagnosis bySCr $\mathrm{GFR}_{-75}$ as surrogates for baseline kidney function was due to inaccurate baseline $\mathrm{SCr}$ estimation in patients with chronic kidney disease $[14,28]$. In contrast, the underestimation of AKI diagnosis when using $\mathrm{SCr}_{\mathrm{ADM}}$ was due to the under-diagnosis of community-acquired AKI [14]. We found most of AKI cases missed by $\mathrm{SCr}_{\mathrm{ADM}}$ occurred within the first 24 hours of ICU admission.

The analysis in a simulated cohort of patients with baseline outpatient SCr showed the higher sensitivity and lower specificity of AKI diagnosis bySCr $\mathrm{GFR}_{\mathrm{GF}}$ in comparison with $\mathrm{SCr}_{\mathrm{ADM}}$. The decision to use $\mathrm{SCr}_{\mathrm{GFR}-75}$ or $\mathrm{SCr}_{\mathrm{ADM}}$ for AKI diagnosis and classification depends on the purpose of the AKI definition. In clinical practice, AKI prevention and prompt treatment might improve patient outcomes. Thus, for risk stratification purposes in clinical practice, we encourage the use of $\mathrm{SCr}_{\mathrm{GFR}-75}$ for AKI diagnosis, as it can conceivably identify more AKI cases. Conversely, for research studies that enroll patients with AKI for invasive tests or treatments, using $\mathrm{SCr}_{\mathrm{ADM}}$ may be more suited, as it would be more likely to enroll patients who are going to benefit from the intervention.

The use of estimated $\mathrm{SCr}$ by back-calculation with the MDRD formula can allow investigators or clinicians to be more flexible regarding the sensitivity and specificity of

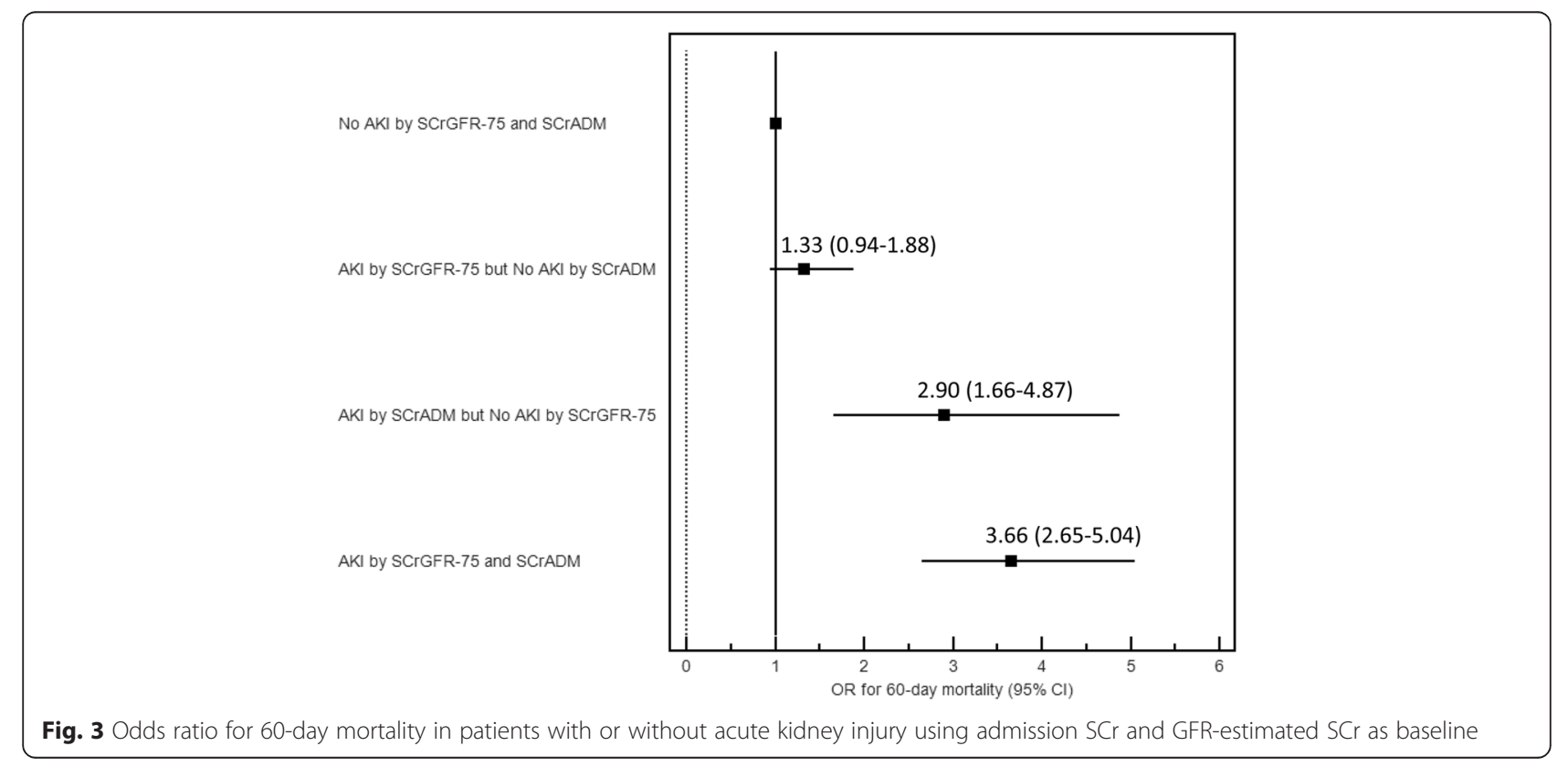


Table 4 The corresponding sensitivities and specificities for AKI diagnosis using an estimated $\mathrm{SCr}$, based on various assumed GFR

\begin{tabular}{|c|c|c|}
\hline $\mathrm{SCr}_{\mathrm{GFR}}\left(\mathrm{ml} / \mathrm{min} / 1.73 \mathrm{~m}^{2}\right)$ & Sensitivity & Specificity \\
\hline 30 & 26.8 & 99.5 \\
\hline 35 & 34.0 & 99.3 \\
\hline 40 & 39.8 & 98.8 \\
\hline 45 & 47.0 & 97.8 \\
\hline 50 & 53.0 & 96.8 \\
\hline 55 & 56.4 & 95.6 \\
\hline 60 & 63.7 & 93.9 \\
\hline 65 & 68.8 & 91.9 \\
\hline 70 & 73.0 & 90.2 \\
\hline 75 & 77.2 & 87.8 \\
\hline 80 & 81.7 & 85.0 \\
\hline 85 & $85.2^{\mathrm{a}}$ & $83.0^{\mathrm{a}}$ \\
\hline 90 & 87.7 & 78.5 \\
\hline 95 & 90.5 & 76.2 \\
\hline 100 & 91.7 & 73.4 \\
\hline 105 & 94.0 & 68.7 \\
\hline 110 & 95.7 & 66.0 \\
\hline 115 & 96.6 & 63.3 \\
\hline 120 & 97.0 & 57.0 \\
\hline $\mathrm{SCr}_{\mathrm{ADM}}$ & 50.5 & 94.8 \\
\hline
\end{tabular}

Abbreviation: $\mathrm{SCr}_{A D M}$ the admission serum creatinine, $\mathrm{SCr}_{G F R}$ GFR-estimated serum creatinine

${ }^{a_{\text {surrogate }}}$ for baseline $\mathrm{SCr}$ yielding highest sum of sensitivity and specificity

the AKI definition. As shown in Table 4 and Additional file 1: Table S3, when using different assumed GFR for SCr estimation, it provides different levels of sensitivity and specificity that we may individualize in each patient in different encounters. Since GFR decreases with age, the use of $\mathrm{SCr}_{\mathrm{GFR}-75}$ might result in over-AKI classification in the elderly [29], therefore use of a different assumed GFR for $\mathrm{SCr}$ estimation could be considered in different age groups. For example, using $\mathrm{SCr}_{\mathrm{GFR}-70}$ in elderly but $\mathrm{SCr}_{\mathrm{GFR}-100}$ in younger adult would yield the highest sum of sensitivity and specificity. In addition, abnormal renal function at presentation may be due to pre-existing chronic kidney disease or due to acute elevation of $\mathrm{SCr}$ from community-acquired acute kidney injury. Therefore, estimated SCr based on lower assumed GFR might be more suitable for patients without baseline outpatient $\mathrm{SCr}$, who present initially with an abnormal SCr. In contrast, using $\mathrm{SCr}_{\mathrm{ADM}}$ in patients with abnormal renal function based on initial $\mathrm{SCr}$ value in hospital would result in lower sensitivity in the diagnosis of AKI.

This study has several limitations. (1) This is a singlecenter retrospective study. (2) We did not include the urine output criterion for AKI diagnosis since an indwelling urinary catheter was not used to obtain accurate hourly urine output data for all critically ill patients. (3) Our study compared only two common surrogates for baseline $\mathrm{SCr}$ and did not include other advanced methods to estimate the baseline SCr. For example, Siew and colleagues recently demonstrated that a multiple imputation method can improve accuracy in estimating missing baseline $\mathrm{SCr}$ and reduce misclassification of AKI [30]. However, the use of this technique in clinical practice is limited and still requires further validation. A multi-center prospective study is ultimately required to address some of these limitations.

\section{Conclusion}

When baseline outpatient $\mathrm{SCr}$ was not available, using $\mathrm{SCr}_{\mathrm{GFR}-75}$ as a surrogate for baseline $\mathrm{SCr}$ was found to be more sensitive but less specific for AKI diagnosis compared to $\mathrm{SCr}_{\mathrm{ADM}}$. This resulted in higher incidence of AKI with larger likelihood of false-positive cases.

\section{Additional file}

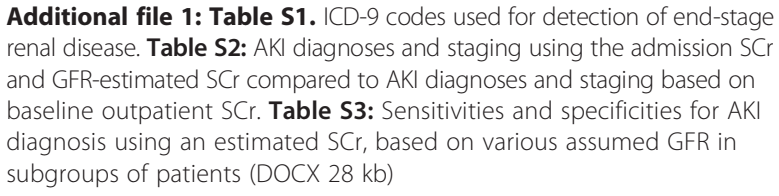

\section{Abbreviations}

AKl: Acute kidney injury; Cl: Confidence interval; GFR: Glomerular filtration rate; ICU: Intensive care unit; IQR: Interquartile range; SCr: Serum creatinine; $\mathrm{SCr}_{\mathrm{ADM}}$ : Admission serum creatinine; $\mathrm{SCr}_{\mathrm{GFR}-75}$ : Estimated serum creatinine, using MDRD formula, based on an assumed glomerular filtration rate of $75 \mathrm{ml} / \mathrm{min} / 1.73 \mathrm{~m}^{2}$.

\section{Competing interests}

The authors declare that they have no competing interests.

\section{Authors' contributions}

CT had full access to the data in the study and takes responsibility for the integrity of the data and the accuracy of the data analysis. CT, WC, and KBK designed the study. CT, WC, WK, PU, and NS collected data. CT, WC, and AMH analyzed and interpreted data, CT, WC, AMH, and KBK drafted and critically revised the manuscript for important intellectual content. KBK supervised all critical parts of the study. All authors read and approved the final manuscript.

\section{Acknowledgements}

The work was supported by Division of Nephrology and Hypertension and Division of Critical Care Medicine, Mayo Clinic. This funding has no role in the design and conduct of the study; collection, analysis and interpretation of the data; or preparation, review and approval of the manuscript.

\section{Author details}

${ }^{1}$ Division of Nephrology and Hypertension, Mayo Clinic, 200 First Street SW, Rochester, MN 55905, USA. ${ }^{2}$ Medical Scientist Training Program, Mayo Clinic, Rochester, MN, USA. ${ }^{3}$ Division of Rheumatology, Mayo Clinic, Rochester, MN, USA. ${ }^{4}$ Division of Pulmonary and Critical Care Medicine, Mayo Clinic, Rochester, MN, USA

Received: 8 February 2015 Accepted: 6 January 2016

Published online: 09 January 2016

\section{References}

1. Hoste EA, Kellum JA, Katz NM, Rosner MH, Haase M, Ronco C. Epidemiology of acute kidney injury. Contrib Nephrol. 2010;165:1-8. 
2. Kashani K, Herasevich V. Sniffing out acute kidney injury in the ICU: do we have the tools? Curr Opin Crit Care. 2013;19(6):531-6.

3. Cheungpasitporn W, Thongprayoon C, Srivali N, O'Corragain OA, Edmonds PJ, Ungprasert $\mathrm{P}$, et al. Preoperative renin-angiotensin system inhibitors use linked to reduced acute kidney injury: a systematic review and meta-analysis. Nephrol Dial Transplant. 2015;30(6):978-88.

4. Thongprayoon C, Cheungpasitporn W, Srivali N, Harrison AM, Gunderson TM, Kittanamongkolchai W, et al. AKI after Transcatheter or Surgical Aortic Valve Replacement. J Am Soc Nephrol 2015. [Epub ahead of print].

5. Chertow GM, Burdick E, Honour M, Bonventre JV, Bates DW. Acute kidney injury, mortality, length of stay, and costs in hospitalized patients. J Am Soc Nephrol. 2005;16(11):3365-70.

6. Ricci Z, Cruz D, Ronco C. The RIFLE criteria and mortality in acute kidney injury: A systematic review. Kidney Int. 2008;73(5):538-46.

7. Ricci Z, Cruz DN, Ronco C. Classification and staging of acute kidney injury: beyond the RIFLE and AKIN criteria. Nat Rev Nephrol. 2011;7(4):201-8.

8. Thongprayoon C, Cheungpasitporn W, Akhoundi A, Ahmed AH, Kashani KB. Actual versus ideal body weight for acute kidney injury diagnosis and classification in critically III patients. BMC Nephrol. 2014;15(1):176.

9. Thongprayoon C, Cheungpasitporn W, Shah IK, Kashyap R, Park SJ, Kashani K, et al. Long-term outcomes and prognostic factors for patients requiring rena replacement therapy after cardiac surgery. Mayo Clin Proc. 2015;90(7):857-64.

10. Thongprayoon C, Cheungpasitporn W, Srivali N, Ungprasert P, Kittanamongkolchai W, Greason KL, et al. Acute kidney injury after transcatheter aortic valve replacement: a systematic review and meta-analysis. Am J Nephrol. 2015;41(4-5):372-82.

11. Kidney Disease: Improving Global Outcomes (KDIGO) Acute Kidney Injury Work Group. KDIGO Clinical Practice Guidelines for Acute Kidney Injury. Kidney Int Suppl. 2012;2(1):1-138.

12. Thongprayoon C, Cheungpasitporn W, Kittanamongkolchai W, Srivali N, Ungprasert P, Kashani K. Optimum methodology for estimating baseline serum creatinine for the acute kidney injury classification. Nephrology (Carlton). 2015;20(12):881-6.

13. Thongprayoon C, Cheungpasitporn W, Srivali N, Ungprasert $P$, Kittanamongkolchai W, Kashani K. The impact of fluid balance on diagnosis, staging and prediction of mortality in critically ill patients with acute kidney injury. J Nephrol. 2015. [Epub ahead of print].

14. Siew ED, Matheny ME, Ikizler TA, Lewis JB, Miller RA, Waitman LR, et al. Commonly used surrogates for baseline renal function affect the classification and prognosis of acute kidney injury. Kidney Int. 2010;77(6):536-42.

15. Bellomo R, Ronco C, Kellum JA, Mehta RL, Palevsky P. Acute renal failure definition, outcome measures, animal models, fluid therapy and information technology needs: the Second International Consensus Conference of the Acute Dialysis Quality Initiative (ADQI) Group. Crit Care. 2004;8(4):R204-212.

16. Pickering JW, Endre ZH. Back-calculating baseline creatinine with MDRD misclassifies acute kidney injury in the intensive care unit. Clin J Am Soc Nephrol. 2010;5(7):1165-73.

17. Zavada J, Hoste E, Cartin-Ceba R, Calzavacca P, Gajic O, Clermont G, et al. A comparison of three methods to estimate baseline creatinine for RIFLE classification. Nephrol Dial Transplant. 2010;25(12):3911-8.

18. Fliser D, Laville M, Covic A, Fouque D, Vanholder R, Juillard L, et al. A European Renal Best Practice (ERBP) position statement on the Kidney Disease Improving Global Outcomes (KDIGO) clinical practice guidelines on acute kidney injury: part 1: definitions, conservative management and contrast-induced nephropathy. Nephrol Dial Transplant. 2012;27(12):4263-72.

19. Hsu CY, Ordonez JD, Chertow GM, Fan D, McCulloch CE, Go AS. The risk of acute renal failure in patients with chronic kidney disease. Kidney Int. 2008;74(1):101-7.

20. Lo LJ, Go AS, Chertow GM, McCulloch CE, Fan D, Ordonez JD, et al. Dialysisrequiring acute renal failure increases the risk of progressive chronic kidney disease. Kidney Int. 2009;76(8):893-9.

21. Singh B, Singh A, Ahmed A, Wilson GA, Pickering BW, Herasevich V, et al. Derivation and validation of automated electronic search strategies to extract Charlson comorbidities from electronic medical records. Mayo Clin Proc. 2012;87(9):817-24.

22. Herasevich V, Pickering BW, Dong Y, Peters SG, Gajic O. Informatics infrastructure for syndrome surveillance, decision support, reporting, and modeling of critical illness. Mayo Clin Proc. 2010;85(3):247-54.

23. Knaus WA, Wagner DP, Draper EA, Zimmerman JE, Bergner M, Bastos PG, et al. The APACHE III prognostic system. Risk prediction of hospital mortality for critically ill hospitalized adults. Chest. 1991;100(6):1619-36.
24. Wentworth DN, Neaton JD, Rasmussen WL. An evaluation of the Social Security Administration master beneficiary record file and the National Death Index in the ascertainment of vital status. Am J Public Health. 1983;73(11):1270-4

25. Gaiao S, Cruz DN. Baseline creatinine to define acute kidney injury: is there any consensus? Nephrol Dial Transplant. 2010;25(12):3812-4.

26. Hoste EA, Clermont G, Kersten A, Venkataraman R, Angus DC, De Bacquer D, et al. RIFLE criteria for acute kidney injury are associated with hospital mortality in critically ill patients: a cohort analysis. Crit Care. 2006;10(3):R73.

27. Candela-Toha AM, Recio-Vazquez M, Delgado-Montero A, del Rey JM, Murie A, Liano F, et al. The calculation of baseline serum creatinine overestimates the diagnosis of acute kidney injury in patients undergoing cardiac surgery. Nefrologia. 2012;32(1):53-8.

28. Bagshaw SM, Uchino S, Cruz D, Bellomo R, Morimatsu H, Morgera S, et al. A comparison of observed versus estimated baseline creatinine for determination of RIFLE class in patients with acute kidney injury. Nephrol Dial Transplant. 2009;24(9):2739-44

29. Spoorenberg SM, Meijvis SC, Navis G, Bos WJW. Age-and genderadjusted eGFR to estimate baseline creatinine for RIFLE criteria. NDT Plus. 2011:4(5):365-6.

30. Siew ED, Peterson JF, Eden SK, Moons KG, Ikizler TA, Matheny ME. Use of multiple imputation method to improve estimation of missing baseline serum creatinine in acute kidney injury research. Clin J Am Soc Nephrol. 2013;8(1):10-8.

\section{Submit your next manuscript to BioMed Central and we will help you at every step:}

- We accept pre-submission inquiries

- Our selector tool helps you to find the most relevant journal

- We provide round the clock customer support

- Convenient online submission

- Thorough peer review

- Inclusion in PubMed and all major indexing services

- Maximum visibility for your research

Submit your manuscript at www.biomedcentral.com/submit
) Biomed Central 